\title{
Management of Maternal Cardiac Arrest in the Third Trimester of Pregnancy: A Simulation-Based Pilot Study
}

\author{
Jacquelyn Adams, ${ }^{1}$ Jose R. Cepeda Brito, ${ }^{2}$ Lauren Baker, ${ }^{1}$ \\ Patrick G. Hughes, ${ }^{2}$ M. David Gothard, ${ }^{3}$ Michele L. McCarroll, ${ }^{4}$ Jocelyn Davis, ${ }^{5}$ \\ Angela Silber, ${ }^{6}$ and Rami A. Ahmed ${ }^{2}$ \\ ${ }^{1}$ Summa Health System, Department of Obstetrics and Gynecology, Akron, OH 44304, USA \\ ${ }^{2}$ Summa Health System, Department of Medical Education, Virtual Care Medical Simulation Laboratory, Akron, \\ OH 44304, USA \\ ${ }^{3}$ Biostats Inc., Canton, OH 44730, USA \\ ${ }^{4}$ Summa Health System, Summa Center for Women's Health Research, Akron, OH 44304, USA \\ ${ }^{5}$ Summa Health System, Department of Nursing Professional Development, Akron, $\mathrm{OH} 44304$, USA \\ ${ }^{6}$ Summa Health System, Department of Obstetrics and Gynecology and Department of Maternal Fetal Medicine, \\ Akron, $\mathrm{OH} 44304, \mathrm{USA}$
}

Correspondence should be addressed to Rami A. Ahmed; ahmedr@summahealth.org

Received 28 January 2016; Accepted 10 July 2016

Academic Editor: Robert Boots

Copyright (C) 2016 Jacquelyn Adams et al. This is an open access article distributed under the Creative Commons Attribution License, which permits unrestricted use, distribution, and reproduction in any medium, provided the original work is properly cited.

Objective. To evaluate confidence, knowledge, and competence after a simulation-based curriculum on maternal cardiac arrest in an Obstetrics \& Gynecologic (OBGYN) residency program. Methods. Four simulations with structured debriefing focusing on high yield causes and management of maternal cardiac arrest were executed. Pre- and post-individual knowledge tests (KT) and confidence surveys (CS) were collected along with group scores of critical performance steps evaluated by content experts for the first and final simulations. Results. Significant differences were noted in individual KT scores (pre: $58.9 \pm 8.9$ versus post: $72.8 \pm 6.1, p=0.01$ ) and CS total scores (pre: $22.2 \pm 6.4$ versus post: $29.9 \pm 3.4, p=0.007$ ). Significant differences were noted in airway management, $p=0.008$; appropriate cycles of drug/shock-CPR, $p=0.008$; left uterine displacement, $p=0.008$; and identifying causes of cardiac arrest, $p=0.008$. Nonsignificant differences were noted for administration of appropriate drugs/doses, $p=0.074$; chest compressions, $p=0.074$; bag-mask ventilation before intubation, $p=0.074$; and return of spontaneous circulation identification, $p=0.074$. Groups remained noncompetent in team leader tasks and considering therapeutic hypothermia. Conclusion. This study demonstrated improved OBGYN resident knowledge, confidence, and competence in the management of third trimester maternal cardiac arrest. Several skills, however, will likely require more longitudinal curricular exposure and training to develop and maintain proficiency.

\section{Introduction}

Cardiac arrest is a rare event during pregnancy [1]. Due to advances in assisted reproductive therapy, the increasing number of pregnancies in women older than thirty-five years of age, and continued intimate partner violence globally [2], the likelihood of maternal cardiac arrest is escalating [3]. Whether cardiac arrest in pregnancy is ultimately attributed to obstetric or nonobstetric etiologies, the cause must be identified in a timely fashion and aggressively managed to improve the likelihood of successful resuscitation of both the mother and fetus. Previous research has shown simulation to be a valuable tool as a means to educate novice providers in the management of emergent obstetrical conditions [4].

Simulation has also been shown to improve competence in obstetric emergency decision-making, leadership, and individual and team performance [5]. This sentiment is reinforced in Committee Opinion Number 590 from The 
American College of Obstetricians and Gynecologists which states that one parameter for preparedness is integration of standardized emergency simulation and drills to identify and correct common clinical errors, to practice effective communication in a crisis, and to reinforce protocols, activation criteria, and critical interventions [6].

The objective of this study was to improve the knowledge, competence, confidence, and crisis resource management (CRM) skills of a team of obstetrical residents in the management of maternal cardiopulmonary arrest through the implementation of a simulation-based curriculum.

\section{Methods}

2.1. Participants and Data Collection. Nine residents from our institution's Obstetrics \& Gynecology (OBGYN) residency program were recruited for the study based on their availability to participate in all four simulations. Residents were evenly assigned to one of two study groups based on their level of training. The residents not part of the study were involved in the educational experience as part of a nonresearch group. Simulations were scheduled during protected time for weekly resident education. The study was considered exempt by the Institutional Review Board.

Participants completed a 7-question confidence survey (CS) utilizing a 5-point Likert scale and a 20-question multiple-choice knowledge test (KT) in a pretest/posttest approach. Competence was assessed by video review of obstetrical residents' management as a team of a simulated maternal cardiac arrest in a similar pretest/posttest fashion. An expert panel composed of a Maternal Fetal Medicine (MFM) attending, an Emergency Medicine (EM) attending, and an Obstetrician/Gynecologist graded the pre- and postintervention simulations using a modified score sheet with items from both TeamSTEPPS ${ }^{\circledR}$ and the American Heart Association ${ }^{\circledR}$ (AHA) Megacode Checklist (see the following checklist).

Resuscitation Checklist for the Management Cardiac Arrest in Pregnancy (Modified from the AHA Megacode Testing Checklist 3)

Critical Performance Steps

Team Leader (Check if Done Correctly)

$\square$ Ensures high-quality CPR at all times.

$\square$ Assigns team member roles.

$\square$ Ensures that team members perform well.

VF Management (Check if Done Correctly)

\author{
$\square$ Recognizes VF. \\ $\square$ Clears before ANALYZE and SHOCK. \\ $\square$ Immediately resumes CPR after shocks. \\ Appropriate airway management.
}

Appropriate cycles of drug-rhythm check/shockCPR.

$\square$ Administers appropriate $\operatorname{drug}(\mathrm{s})$ and doses.

BLS/ACLS Modifications for Pregnancy (Check if Done Correctly)

$\square$ Activates protocol for an emergency cesarean delivery as soon as cardiac arrest is identified.

Performs manual left uterine displacement.

$\square$ Performs chest compressions slightly higher on the sternum than normally recommended.

$\square$ Uses bag-mask ventilation with $100 \% \mathrm{O}_{2}$ before intubation is done.

$\square$ Attempts to identify common and reversible causes of cardiac arrest in pregnancy.

$\square$ Delivers infant by emergency cesarean section.

$\square$ Delivers infant no more than 5 minutes after cardiac arrest ensues.

Postcardiac Arrest (Check if Done Correctly)

$\square$ Identifies ROSC.

$\square$ Ensures BP and 12-lead ECG are performed and $\mathrm{O}_{2}$ saturation is monitored, verbalizes need for endotracheal intubation and waveform capnography, and orders laboratory tests.

$\square$ Considers therapeutic hypothermia.

2.2. Curriculum Design. The medical simulation staff, two senior level OBGYN residents, and the Director of the MFM department at our institution jointly designed a fourcase simulation based curriculum. Educational objectives were modeled after the current AHA guidelines for the management of maternal cardiac arrest (see Figure 1) and the TeamSTEPPS CRM course [7, 8].

All simulations were based on presentations that could lead to maternal cardiac arrest [9]. The four scenario stems were massive pulmonary embolism, magnesium toxicity, placental abruption due to motor vehicle accident, and blunt abdominal trauma caused by intimate partner violence. Data collection was performed prior to the first and final simulations. This data collection process included a confidence survey, knowledge test, and evaluation of the team simulation scenarios by the faculty content experts. No evaluation was performed for the intervention phase simulation scenarios. During the intervention phase residents managed two additional cases utilizing a deliberate practice approach [10]. All four simulations were followed by faculty led debriefing on team performance by our panel of content experts. The knowledge test and confidence surveys were not collected for the intervention phase. All data collected was prior to and following the intervention phase. Videos were reviewed and scored by content experts/faculty of the first summative simulation and the final (4th) summative simulation. 


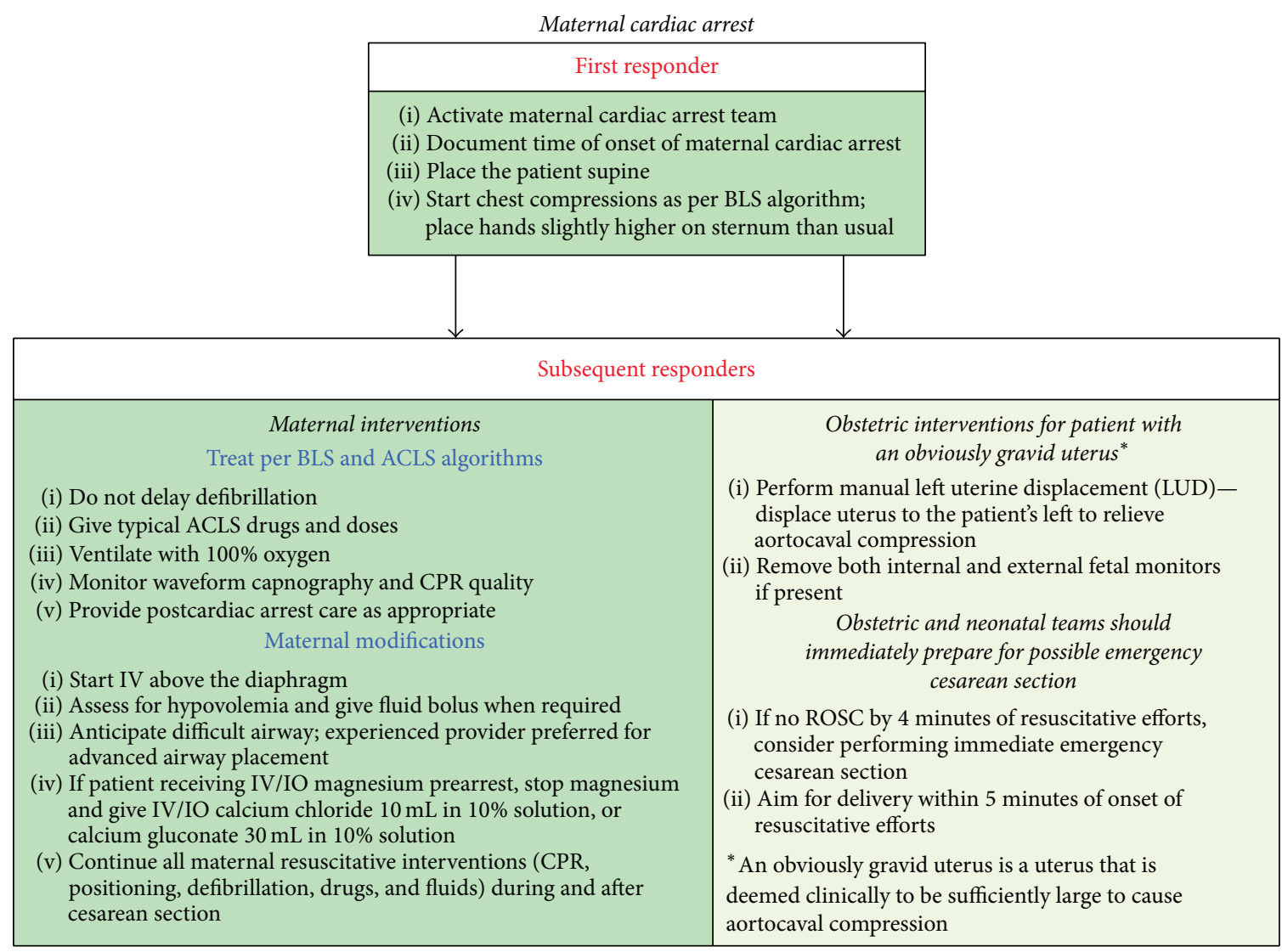

Search for and treat possible contributing factors
(BEAU-CHOPS)
Bleeding/DIC
Embolism: coronary/pulmonary/amniotic fluid embolism
Anesthetic complications
Uterine atony
Cardiac disease (MI/ischemia/aortic dissection/cardiomyopathy)
Hypertension/preeclampsia/eclampsia
Other: differential diagnosis of standard ACLS guidelines
Placenta abruptio/previa
Sepsis $\quad$ C) 2010 American Heart Association

FIGURE 1: Maternal cardiac arrest algorithm.

2.3. Materials and Models. All simulations were performed in the simulation laboratory of Summa Health System. A confederate nurse and real time patient feedback were used in all simulations to facilitate information gathering and scenario flow. A digital monitor displaying dynamic vital signs was readily available and modified based on case progression. Cases were ended at the faculty member's discretion once 10 minutes after maternal cardiac arrest had elapsed.

Gaumard's NOELLE Maternal and Neonatal Birthing Simulator was used for all simulations. To enable the performance of an emergent perimortem cesarean section, the simulator was fitted with a disposable abdominal wall and amniotic sac unit. The amniotic sac was emulated by a red biohazard bag, the NOELLE infant model (basic), water, and food coloring. The simulator electrical and mechanical systems were deactivated and protected by plastic liners. The amniotic sac unit was placed in the abdomen of the NOELLE simulator. This unit was then covered by flank steak to simulate the fascia and muscle layers of the abdomen. These two layers were then protected by two-inch model foam to serve as the subcutaneous fat. To hold the layers of the abdomen in place, an iodoform band was attached from the pelvis to the breast line of the model. The skin was recreated by flesh-colored duct tape keeping the abdominal layers secure (see Figure 2). A standard crash cesarean section kit was available upon request from the resuscitation team 


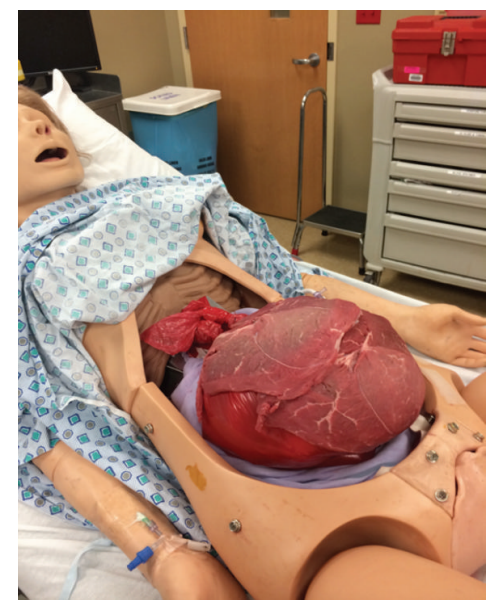

(a)

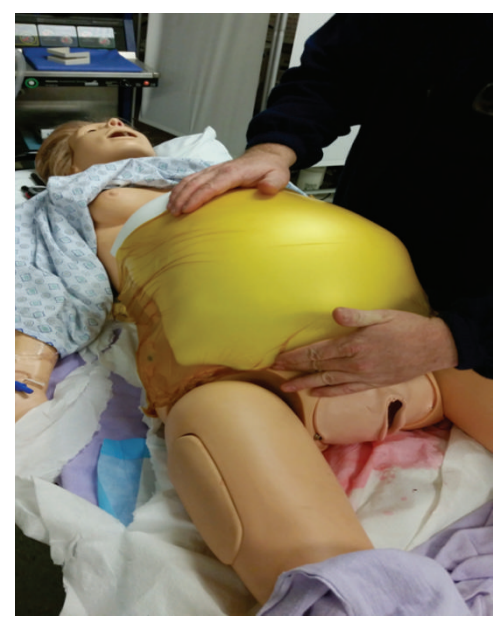

(c)

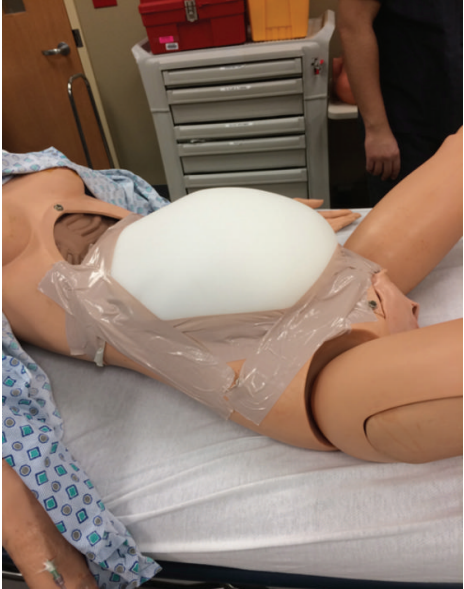

(b)

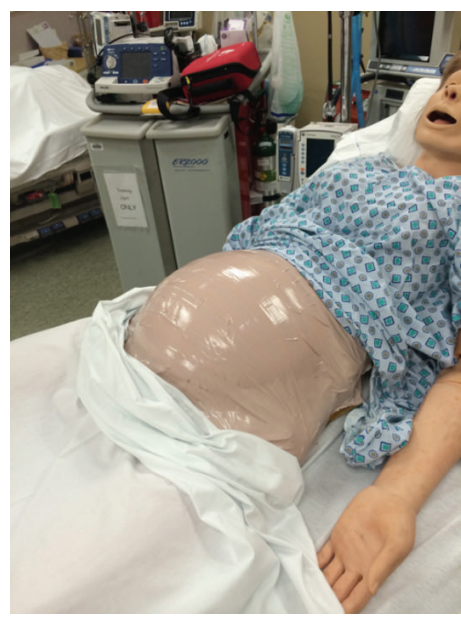

(d)

Figure 2: Modified Gaumard’s NOELLE ${ }^{\circledR}$ Maternal and Neonatal Birthing Simulator. (a) Disposable abdominal wall and amniotic sac unit covered by flank steak, emulating fascia, and muscle layers. (b) Subcutaneous fat later made from two-inch model foam. (c) Internal layers held in place by an iodoform band. (d) Skin recreated from flesh-colored duct tape.

to allow for performance of emergent perimortem cesarean section.

2.4. Data Analysis. Individual CS, KT, and simulation team performance scores were analyzed using SPSS 22.0.

\section{Results}

Resident participation was $45 \%$ (9 of 20). Average participant age was $29.6 \pm 1.1$ years $(n=9)$. Significant differences were noted in individual KT scores (pre: $58.9 \pm 8.9$ versus post: $72.8 \pm 6.1, p=0.01$ ) and CS total scores (pre: $22.2 \pm 6.4$ versus post: $29.9 \pm 3.4, p=0.007$ ). Significant differences were noted in group competencies of airway management, $p=0.008$; appropriate cycles of drug shock-CPR, $p=0.008$; left uterine displacement, $p=0.008$; and identifying causes of cardiac arrest in pregnancy, $p=0.008$. Nonsignificant differences were noted after intervention on the residents' competencies for administration of appropriate drugs/doses, $p=0.074$; chest compressions, $p=0.074$; bag-mask ventilation before intubation, $p=0.074$; and identification of return of spontaneous circulation identification, $p=0.074$. Groups remained noncompetent in providing high-quality cardiopulmonary resuscitation at all times, team leader assigning roles, or considering therapeutic hypothermia. See Tables 1 and 2 for results.

\section{Discussion}

In our study we noted a significant improvement in the critical performance steps of airway management, adherence to advanced cardiovascular life support (ACLS) treatment algorithm, manual left uterine displacement, and identification of common causes of maternal cardiac arrest. There was also improvement of KT scores from baseline. This trend was also demonstrated in the increase in CS from the initial evaluation to the postintervention stage. This is likely a result of the focused, interactive learning environment afforded in 
TABLE 1: Residents confidence and knowledge scores before and after intervention.

\begin{tabular}{lccc}
\hline Test scores & Before intervention & After intervention & Change in score \\
\hline Knowledge (\%) & & & $13.9(11.93)$ \\
Mean (SD) & $58.9(8.94)$ & $72.8(6.18)$ & 10 \\
Median & 65 & 75 & $0-35$ \\
Min-Max & $45-70$ & $65-80$ & \\
\hline Self-reported confidence & & & 7.016 \\
Mean (SD) & $22.2(6.42)$ & $29.9(3.41)$ & $7.82)$ \\
Median & 24 & 32 & 0.007 \\
\hline
\end{tabular}

Note: ${ }^{*} p$ value via Wilcoxon signed rank test.

the simulation laboratory and the expert debriefing following all simulations.

Cardiopulmonary arrest in pregnancy is a high-risk, low frequency scenario and as such requires continued training and preparation to maintain an appropriate skill level to manage these complex patients [11-13]. The use of simulation to improve outcomes has been demonstrated in multiple arenas because it gives the learner the opportunity to experience uncommon scenarios, receive feedback, and perhaps most importantly correct mistakes before they become part of the learner's or group's mental model [14].

Despite the training intervention, groups did not demonstrate a significant improvement in team leader specific tasks of ensuring high-quality cardiopulmonary resuscitation (e.g., minimizing disruptions in chest compression) and role assignment. During the debriefing sessions team leaders expressed difficulty assigning roles and determining a team leader because, in some instances, there were multiple senior level residents in the teams. This varies greatly from their normal call schedule. We used this opportunity to emphasize the importance of having a clearly defined leader, how to choose a team leader among peers, and how those "would be leaders" can be an asset to the team by being the first follower [15]. We also emphasized the importance of closedloop communication during the resuscitation to improve team communication and avoid management errors [16].

The critical performance steps that did not show improvement, pertaining to CRM (e.g., team leadership), likely need continued longitudinal training throughout residency to achieve competence. Consistent with current ACLS training literature regarding skill decay, our belief is that the lack of consideration of therapeutic hypothermia, as well as the areas where residents remained noncompetent, was likely due to the expected decline in knowledge and psychomotor skill retention after ACLS certification [17].

Residents performed a perimortem cesarean section during all simulations. However, we observed an unexpected trend with almost all groups choosing to perform a Pfannenstiel skin incision and a low transverse uterine incision, contrasting with the current practice guidelines that favor a midline vertical incision (see Figure 3). Residents reported feeling they would be more proficient and could expedite delivery using this approach. This decision-making process has been supported in the literature by other authors [18, 19]. Group debriefing highlighted the advantages of a midline abdominal incision particularly for cases of abdominal

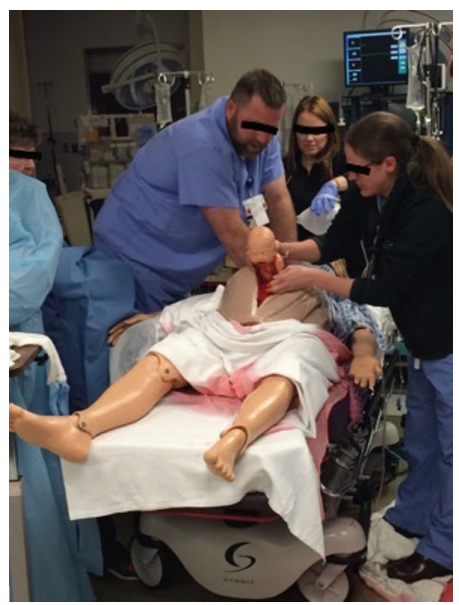

FIGURE 3: Residents performing emergent perimortem cesarean section.

trauma, where general surgery may need to be involved in the resuscitative efforts.

Our study had several limitations including a small sample size and residents from one concentration (obstetrics and gynecology) from a single institution. We hope to further strengthen our simulation-based curriculum by providing a true interprofessional learning environment. In future simulation scenarios, ideally we would include multiple disciplines and levels of training to help the assignment of team roles and execution of tasks feel more appropriate for the learner. Based on our study, further practice with these scenarios is needed to solidify the important concepts pertaining to cardiovascular collapse. In our institution, we plan to do this by utilizing these scenarios starting with the incoming interns' initial "boot camp" and repeating this training through all four years to track progress, decrease skill decay, and solidify concepts with which the learner may not be regularly faced. For other groups attempting a similar study or for obstetrics and gynecology learners in general, our project demonstrated the need to enforce early and often the key changes for maternal cardiac arrest management. It also demonstrated that further training and enforcement of team leader skills is crucial to improve the confidence and performance of the team. 
TABLE 2: Residents team competence scores (paired) before and after intervention.

\begin{tabular}{|c|c|c|c|c|}
\hline \multirow{2}{*}{\multicolumn{2}{|c|}{ Critical performance steps (area of competency/posttest competency) }} & \multicolumn{3}{|c|}{ Pretest competency } \\
\hline & & \multicolumn{3}{|c|}{ Not competent Competent ${ }^{\wedge} p$ value $^{\&}$} \\
\hline \multicolumn{5}{|l|}{ Team leader } \\
\hline \multirow{2}{*}{ Ensures high-quality CPR at all times } & Not Competent & $9(100 \%)$ & & NA \\
\hline & Competent & & & \\
\hline \multirow{2}{*}{ Assigns team member roles } & Not Competent & $9(100 \%)$ & & NA \\
\hline & Competent & & & \\
\hline \multirow{2}{*}{ Ensures that team members perform well } & Not Competent & $5(55.6 \%)$ & & 0.134 \\
\hline & Competent & $4(44.4 \%)$ & & \\
\hline \multicolumn{5}{|l|}{ VF management } \\
\hline \multirow{2}{*}{ Recognizes VF } & Not Competent & $5(55.6 \%)$ & & 0.134 \\
\hline & Competent & $4(44.4 \%)$ & & \\
\hline \multirow{2}{*}{ Clears before ANALYZE and SHOCK } & Not Competent & & & NA \\
\hline & Competent & & $9(100 \%)$ & \\
\hline \multirow{2}{*}{ Immediately resumes CPR after shocks } & Not Competent & & & NA \\
\hline & Competent & & $9(100 \%)$ & \\
\hline \multirow{2}{*}{ Appropriate airway management } & Not Competent & & & 0.008 \\
\hline & Competent & $9(100 \%)$ & & \\
\hline \multirow{2}{*}{ Appropriate cycles of drug-rhythm check/shock-CPR } & Not Competent & & & 0.008 \\
\hline & Competent & $9(100 \%)$ & & \\
\hline \multirow{2}{*}{ Administer appropriate $\operatorname{drug}(\mathrm{s})$ and doses } & Not Competent & $9(100 \%)$ & & NA \\
\hline & Competent & & & \\
\hline \multicolumn{5}{|l|}{ BLS/ACLS modifications for pregnancy } \\
\hline \multirow{2}{*}{$\begin{array}{l}\text { Activates protocol for an emergency cesarean delivery as soon as cardiac } \\
\text { arrest is identified }\end{array}$} & Not Competent & & & 0.134 \\
\hline & Competent & $4(44.4 \%)$ & $5(55.6 \%)$ & \\
\hline \multirow{2}{*}{ Positions patient in left-lateral tilt or performs manual uterine displacement } & Not Competent & & & 0.008 \\
\hline & Competent & $9(100 \%)$ & & \\
\hline \multirow{2}{*}{$\begin{array}{l}\text { Performs chest compressions slightly higher on sternum than normally } \\
\text { recommended }\end{array}$} & Not Competent & & & 0.074 \\
\hline & Competent & $5(55.6 \%)$ & $4(44.4 \%)$ & \\
\hline \multirow{2}{*}{ Uses bag-mask ventilation with $100 \% \mathrm{O}_{2}$ before intubation is done } & Not Competent & & & 0.074 \\
\hline & Competent & $5(55.6 \%)$ & $4(44.4 \%)$ & \\
\hline \multirow{2}{*}{$\begin{array}{l}\text { Attempts to identify common and reversible causes of cardiac arrest in } \\
\text { pregnancy }\end{array}$} & Not Competent & & & 0.008 \\
\hline & Competent & $9(100 \%)$ & & \\
\hline \multirow{2}{*}{ Delivers infant by emergency cesarean section } & Not Competent & & & NA \\
\hline & Competent & & $9(100 \%)$ & \\
\hline \multirow{2}{*}{ Delivers infant no more than 5 minutes after cardiac arrest ensues } & Not Competent & & & 0.074 \\
\hline & Competent & $5(55.6 \%)$ & $4(44.4 \%)$ & \\
\hline \multicolumn{5}{|l|}{ Postcardiac arrest } \\
\hline \multirow{2}{*}{ Identifies ROSC } & Not Competent & & & 0.074 \\
\hline & Competent & $5(55.6 \%)$ & $4(44.4 \%)$ & \\
\hline $\begin{array}{l}\text { Ensures BP and } 12 \text { lead ECG are performed and } \mathrm{O}_{2} \text { saturation is monitored, } \\
\text { verbalizes need for endotracheal intubation and waveform capnography, } \\
\text { and orders laboratory test }\end{array}$ & $\begin{array}{l}\text { Not Competent } \\
\text { Competent }\end{array}$ & $9(100 \%)$ & & NA \\
\hline Considers therapeutic hypothermia & Not Competent & $9(100 \%)$ & & NA \\
\hline Considers therapeutic hypothermıa & Competent & & & \\
\hline
\end{tabular}

Note: ${ }^{\&} p$ value via McNemar's test. ${ }^{\wedge}$ Competency is defined by a majority of rater's competency determination. 


\section{Conclusion}

This simulation-based study demonstrated improved OBGYN resident knowledge, confidence, and competence in several areas of the management of third trimester maternal cardiac arrest. Several skills, however, will likely require more longitudinal curricular exposure and training to develop and maintain proficiency.

$\begin{array}{ll}\text { Abbreviations } \\ \text { CRM: } & \text { Crisis resource management } \\ \text { OBGYN: } & \text { Obstetrician/Gynecologist } \\ \text { CS: } & \text { Confidence survey } \\ \text { KT: } & \text { Knowledge test } \\ \text { MFM: } & \text { Maternal Fetal Medicine } \\ \text { EM: } & \text { Emergency Medicine } \\ \text { AHA: } & \text { American Heart Association } \\ \text { CPR: } & \text { Cardiopulmonary resuscitation } \\ \text { ACLS: } & \text { Advanced Cardiac Life Support. }\end{array}$

\section{Competing Interests}

There is no conflict of interests regarding the publication of this paper.

\section{Authors' Contributions}

(i) Study conception and design were done by Jacquelyn Adams, Jose R. Cepeda Brito, Lauren Baker, Patrick G. Hughes, M. David Gothard, Michele L. McCarroll, Jocelyn Davis, Angela Silber, and Rami Ahmed. (ii) Acquisition of data was done by Jacquelyn Adams, Jose R. Cepeda Brito, Lauren Baker, Patrick G. Hughes, Michele L. McCarroll, and Rami Ahmed. (iii) Analysis and interpretation of data were done by Jacquelyn Adams, Jose R. Cepeda Brito, M. David Gothard, Michele L. McCarroll, and Rami Ahmed. (iv) Drafting of the paper was done by Jacquelyn Adams, Jose R. Cepeda Brito, Michele L. McCarroll, Jocelyn Davis, and Rami Ahmed. (v) Critical revision was done by Jacquelyn Adams, Jose R. Cepeda Brito, Lauren Baker, Patrick G. Hughes, M. David Gothard, Michele L. McCarroll, Jocelyn Davis, Angela Silber, and Rami Ahmed.

\section{Acknowledgments}

Several people were instrumental in the completion of this pilot study and the authors would like to acknowledge them here. They would like to thank the staff of the Virtual Care Simulation Laboratory, especially S. Scott Atkinson, for his role in the development of the modifications to the birthing simulator so that the participants could perform a life-like cesarean section and Alma Benner for her technical support during all the simulations. They would also like to thank the Akron City Hospital's Labor and Delivery nursing staff for their role as support staff during all the simulations, and Jennifer Frey for her valuable guidance through the submission and preparation process. Finally, they would like to thank their faculty, Dr. Edward Ferris, Dr. Thomas
Mendise, and Dr. Susan Shondel who very graciously donated their time to further their residents' education by serving as content experts.

\section{References}

[1] S. E. King and D. A. Gabbott, "Maternal cardiac arrest-rarely occurs, rarely researched," Resuscitation, vol. 82, no. 7, pp. 795796, 2011.

[2] J. McFarlane, J. Maddoux, S. Cesario et al., "Effect of abuse during pregnancy on maternal and child safety and functioning for 24 months after delivery," Obstetrics \& Gynecology, vol. 123, no. 4, pp. 839-847, 2014.

[3] C. Montufar-Rueda and A. Gei, "Cardiac arrest during pregnancy," Clinical Obstetrics and Gynecology, vol. 57, no. 4, pp. 871881, 2014

[4] S. Maslovitz, G. Barkai, J. B. Lessing, A. Ziv, and A. Many, "Recurrent obstetric management mistakes identified by simulation," Obstetrics \& Gynecology, vol. 109, no. 6, pp. 1295-1300, 2007.

[5] B. Robertson, L. Schumacher, G. Gosman, R. Kanfer, M. Kelley, and M. DeVita, "Simulation-based crisis team training for multidisciplinary obstetric providers," Simulation in Healthcare, vol. 4, no. 2, pp. 77-83, 2009.

[6] "Committee opinion no. 590: preparing for clinical emergencies in obstetrics and gynecology," Obstetrics \& Gynecology, vol. 123, no. 3, pp. 722-725, 2014.

[7] T. L. Vanden Hoek, L. J. Morrison, M. Shuster et al., "Part 12: cardiac arrest in special situations: 2010 american heart association guidelines for cardiopulmonary resuscitation and emergency cardiovascular care," Circulation, vol. 122, no. 18, supplement 3, pp. S829-S861, 2010.

[8] F. Sheppard, M. Williams, and V. R. Klein, "TeamSTEPPS and patient safety in healthcare," Journal of Healthcare Risk Management, vol. 32, no. 3, pp. 5-10, 2013.

[9] Royal College of Obstetricians and Gynaecologists, Green top guideline no. 56. Maternal collapse in pregnancy and the puerpurium, January 2011.

[10] K. A. Ericsson, "Acquisition and maintenance of medical expertise: a perspective from the expert-performance approach with deliberate practice," Academic Medicine, vol. 90, no. 11, pp. 14711486, 2015.

[11] I. Hayes, R. Rathore, and K. Enohumah, "Prevalence of factors associated with difficult intubation in early and late pregnancy: a prospective observational study," Anaesthesia, vol. 66, pp. 234235, 2011.

[12] S. Lipman, K. Daniels, B. Carvalho et al., "Deficits in the provision of cardiopulmonary resuscitation during simulated obstetric crises," Obstetric Anesthesia Digest, vol. 31, no. 3, pp. 173-174, 2011.

[13] V. Bogne, C. Kirkpatrick, and Y. Englert, "Simulation training in the management of obstetric emergencies. A review of the literature," Revue Medicale de Bruxelles, vol. 35, no. 6, pp. 491498, 2014.

[14] M. Green, C. Rider, D. Ratcliff, and B. C. Woodring, "Developing a systematic approach to obstetric emergencies," Journal of Obstetric, Gynecologic, \& Neonatal Nursing, vol. 44, no. 5, pp. 677-682, 2015.

[15] D. Raffo, "Teaching followership in leadership education," Journal of Leadership Education, vol. 12, no. 1, pp. 262-273, 2013. 
[16] H. Prabhakar, J. B. Cooper, A. Sabel, S. Weckbach, P. S. Mehler, and P. F. Stahel, "Introducing standardized 'readbacks' to improve patient safety in surgery: a prospective survey in 92 providers at a public safety-net hospital," BMC Surgery, vol. 12, no. 1, article 8, 2012.

[17] C.-W. Yang, Z.-S. Yen, J. E. McGowan et al., "A systematic review of retention of adult advanced life support knowledge and skills in healthcare providers," Resuscitation, vol. 83, no. 9, pp. 10551060, 2012.

[18] N. J. McDonnell, "Cardiopulmonary arrest in pregnancy: two case reports of successful outcomes in association with perimortem Caesarean delivery," British Journal of Anaesthesia, vol. 103, no. 3, pp. 406-409, 2009.

[19] V. L. Katz, "Perimortem cesarean delivery: its role in maternal mortality," Seminars in Perinatology, vol. 36, no. 1, pp. 68-72, 2012. 


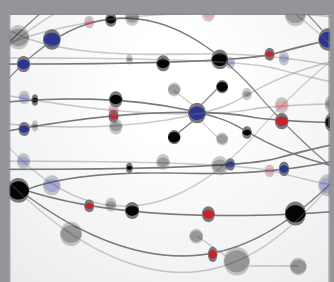

The Scientific World Journal
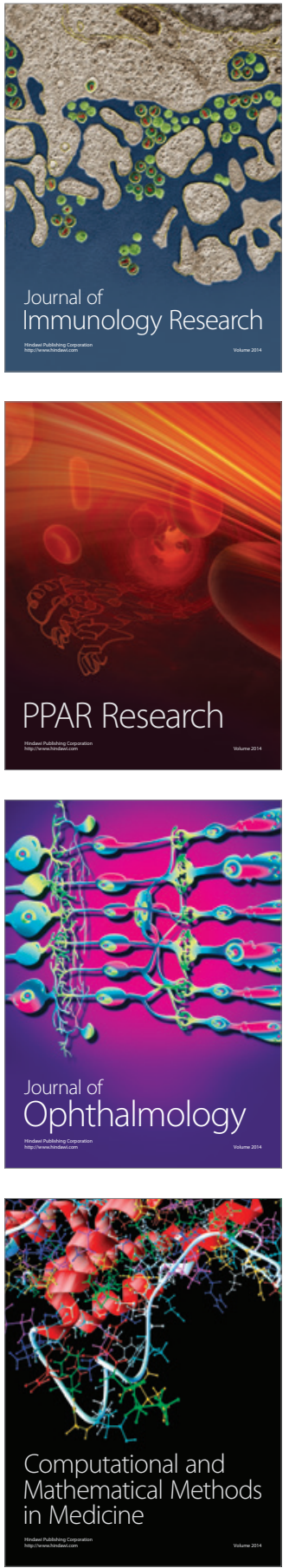

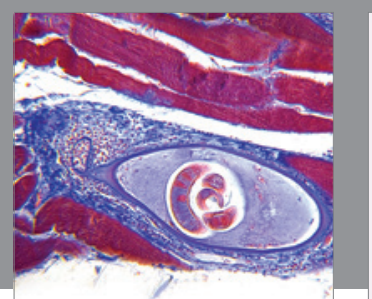

Gastroenterology Research and Practice

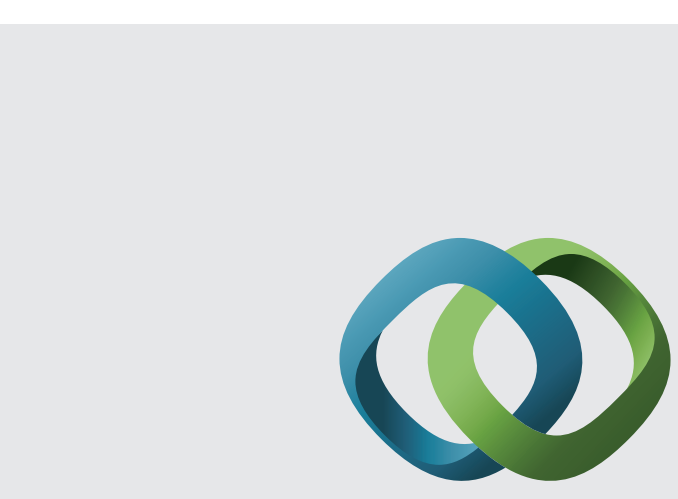

\section{Hindawi}

Submit your manuscripts at

http://www.hindawi.com
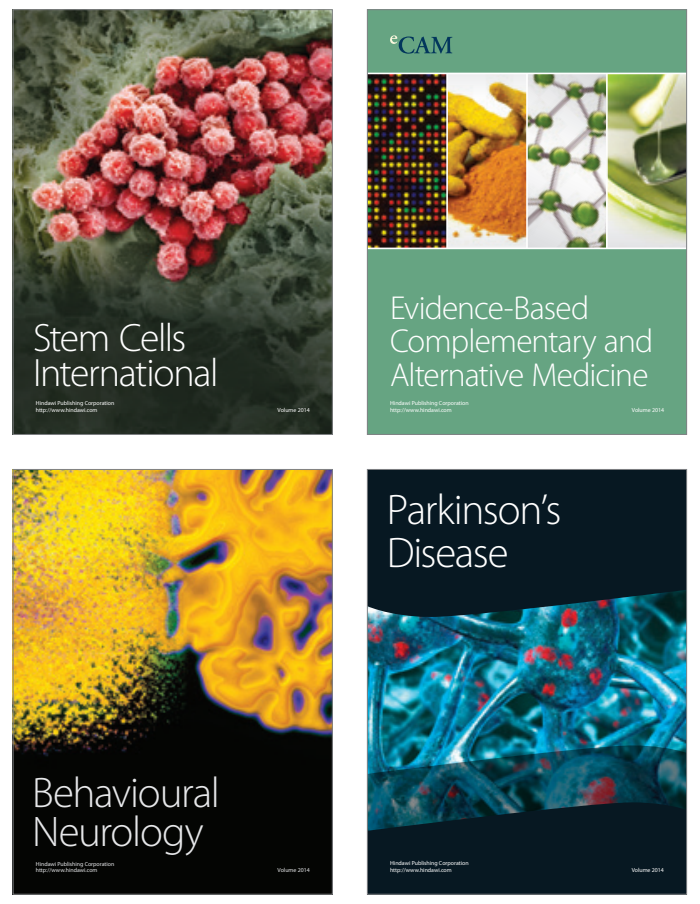
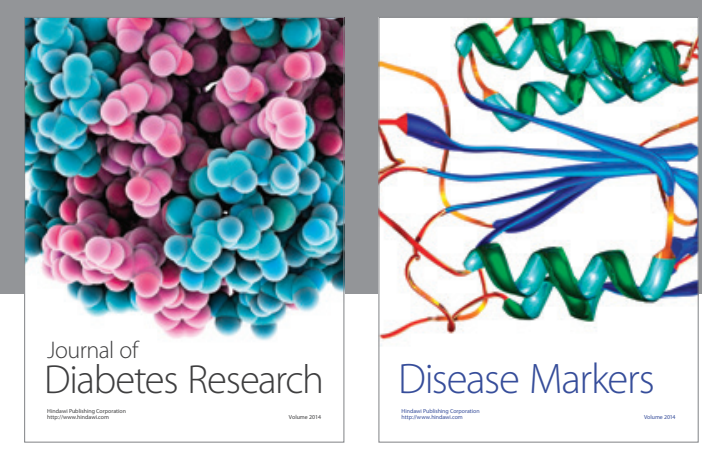

Disease Markers
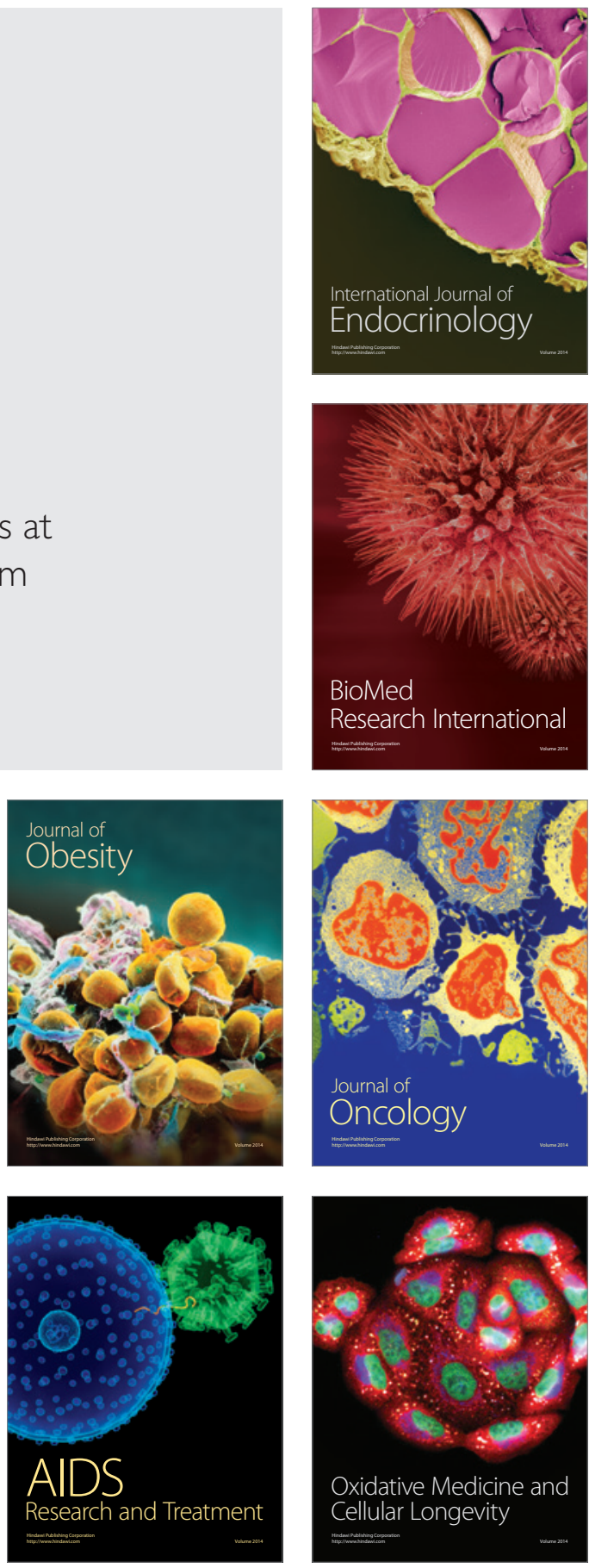